\title{
Le RetOur au DÉSERT DE \\ Catherine Marnas ${ }^{1}$
}

\section{Guillaume Pinçon}

Comme l'indique son titre, le colloque Koltès, maintenant cherche à mettre en relation l'œuvre de Bernard-Marie Koltès avec la période actuelle. Évidemment, c'est au théâtre que ce rapport s'exprime de la manière la plus efficace. La mise en scène dont il sera question est particulièrement pertinente de ce que peut dire du monde d'aujourd'hui une pièce comme Le Retour au désert remise au jour par Catherine Marnas vingt ans après sa publication et présentée en novembre 2008 au Théâtre de la Ville, représentation-référence de cet exposé. Il s'agira ainsi de voir en quoi ce spectacle actualise l'œuvre de départ, la remet à jour et prend acte du monde d'aujourd'hui, dans une problématique du rapport à l'étranger et à l'autre.

Il est d'ailleurs significatif que le drame du Retour au désert se déclenche dès l'arrivée du personnage de Mathilde dans une maison à laquelle elle est a priori étrangère. Revenue d'Algérie, de l'étranger donc, elle rapporte dans ses valises les conflits passés avec son frère Adrien et avec la ville qui l'a bannie. Elle annonce ainsi la couleur dès la deuxième scène: "Je ne fuis aucune guerre; je viens au contraire la porter ici, dans cette bonne ville...”* D'un conflit particulier et personnel, on passe ainsi à la guerre d'Algérie à laquelle il est fait référence et dont l'affrontement est introduit à l'intérieur de la maison française par le retour de Mathilde et, avec elle, de traces de l'ailleurs algérien: la langue arabe dans laquelle s'exprime d'emblée ce personnage et le prénom de sa fille, Fatima. Plus généralement le ressort dramatique repose sur la confrontation de cette double altérité - qui en plus est ouverte au monde, Mathilde se référant à d'autres villes étrangères: Hong Kong, Bamako, Amecameca $^{*}$ à un territoire fermé entre ses murs, cherchant à s'exclure de l'extérieur comme l'exprime consciemment le personnage d'Adrien, par exemple, scène 4: "Le monde est ici, mon fils, [...] et il n'y a rien d'autre à connaître.”* Il s'agit ainsi d'une maison-

* (KOLTÈS, Bernard-Marie. Le Retour au désert. Paris: Minuit, 1988: 13.)

"(Ibidem: 16.)

${ }^{1}$ Ce texte a été écrit à l'occasion du colloque Koltès, maintenant organisé par Christophe Bident, Arnaud Maisetti et Christophe Triau. Celui-ci s'est déroulé du 12 au 14 novembre 2009 à l'université Paris 7 Denis Diderot. 
(Ibidem: 15.)

(Ibidem: 28.)

- (KOLTÈS, Bernard-Marie Lettres. Paris: Minuit, 2009.)

- (KOLTÈS, Bernard-Marie Le Retour au désert. op. cit.: 9.) monde et celle-ci se trouve menacée par l'introduction de l'autre, Mathilde, et de l'ailleurs, les pays étrangers. Mais cette confrontation est paradoxale puisqu' intrinsèque à ce territoire, comme l'exprime Adrien lorsqu'il dit à Mathilde, toujours dans la deuxième scène: "Tiens-toi dans ta propre maison comme une invitée". . L'affrontement à l'autre se joue donc sur plusieurs plans, sur plusieurs territoires et Le Retour au désert en présente les conséquences dramatiques multiples: l'envie de voir l'ailleurs de Mathieu, la fragilisation du territoire propre d'Adrien, la phobie réactionnaire de Plantières exprimée en scène 5 , lors de la réalisation de la vengeance de Mathilde: "Devrai-je avoir peur, bientôt, de violences dans ma propre maison?"”

La dynamique dramatique du Retour au désert est ainsi animée par la confrontation du propre et de l'autre, dynamique que l'on pourrait trouver également dans d'autres pièces de Koltès. S'il s'agirait bien là d'un motif récurrent, on pourrait le justifier par les nombreux voyages que Koltès a faits durant sa vie et dont on a quelques traces dans le recueil de ses Lettres* publié cette année. Dans cette vie en pointillés alternent ainsi des allers et des retours entre des territoires propres, Pralognan par exemple, et des territoires autres - le Brésil, le Mexique, New York, etc., pendant lesquels il met en œuvre l'écriture de nombre de ses pièces. Exemple particulièrement significatif, l'écriture de Combat de nègres et de chiens s'est faite dans sa majeure partie au Guatemala où il restera quelques mois en 1978, succédant la même année à un voyage au Nigéria dont on devine le lien essentiel avec cette pièce qu'il publiera deux ans plus tard. Dans Le Retour au désert, Koltès imagine la présence d'une altérité à l'intérieur d'une "ville de province, à l'est de la France", " première didascalie de la pièce, dont on devine qu'il fait référence à un territoire propre, Metz, où il vécut durant son enfance. Le face à face du propre et de l'autre semble ainsi constituer une dynamique plus générale qui s'exprime à travers ses œuvres. Un "sens du monde", pour reprendre l'expression de Christophe Bident $^{2}$, qui irait ainsi en direction de l'étranger, de l'autre.

La mise en scène du Retour au désert doit beaucoup à un tel "sens du monde". D'abord parce qu'il est caractéristique du parcours personnel et professionnel de Catherine Marnas, particulièrement marqué par sa rencontre avec le Mexique, constituant

\footnotetext{
${ }^{2}$ Et qui donne le titre de la communication qu'il a présentée dans le du colloque Koltès, mentionnée ci-dessus.
} 
dans sa biographie un véritable moment d'épiphanie. C'est dans ce pays qu'elle mit en scène pour la première fois une pièce de Koltès, Roberto Zucco, en 1995. S'il s'agissait de prime abord d'une volonté de faire découvrir ce dramaturge à un pays qui le connaissait peu, cette mise en scène à l'étranger permit surtout de révéler "dans cette pièce et chez Koltès plus généralement un rapport à la mort devenu évident lors de la création et des représentations de Roberto Zucco où cette thématique prenait face au public mexicain une force très compréhensible." 3 Au regard du parcours de Catherine Marnas, de manière plus générale, on trouve ainsi deux récurrences singulières qui se mettent en parallèle: $d$ 'une part, de nombreux voyages à l'étranger par le biais d'activités théâtrales (en Chine ou au Cambodge, par exemple); d'autre part, les nombreux travaux qu'elle a menés à partir de l'œuvre de Koltès. De ce parallèle, ou de ce croisement, la mise en scène de Retour au désert est particulièrement caractéristique puisqu'elle a été créée au Brésil en 2008 au festival de théâtre São José do Rio Preto dans l'état de São Paulo, que s'y croisent le texte original de Retour au désert et sa traduction en portugais du Brésil par Angela Leite Lopes, et enfin qu'y cohabitent les comédiens permanents de la compagnie de Catherine Marnas avec des comédiens brésiliens rencontrés sur place. Cette caractéristique franco-brésilienne de la mise en scène fait ainsi écho de manière particulièrement pertinente au texte de Koltès dans la mesure où s'y trouvent confrontés également des langues et des nationalités.

C'est d'abord en ce sens que le Retour au désert de Catherine Marnas actualise sur scène les confrontations dramatiques de l'œuvre originale. Toutefois, de la même manière que dans le texte celles-ci se jouent sur plusieurs plans et au sein de chaque personnage, de même dans le spectacle il s'agit de multiplier les confrontations et de ne pas s'en tenir à la première franco-brésilianité. De fait, il n'y a pas de partition systématique sur scène entre les comédiens français et brésiliens, même si on peut remarquer au départ leur appartenance à tel ou tel pays. En effet, et c'est là une caractéristique du travail de direction de Catherine Marnas, les comédiens et leurs façons de jouer sont tous volontairement différents. Ce qu'elle recherche plus précisément, c'est ce qu'elle appelle la

\footnotetext{
${ }^{3}$ Discours rapporté d'un entretien avec Catherine Marnas qui eut lieu en août 2009, à Marseille. Les autres citations de cet article sans appel de notes sont également issues de cet entretien.
} 
- (KOLTÈS, Bernard-Marie. Le Retour au désert. op. cit.: 86.) transparence, c'est-à-dire "la manière dont un personnage laisse voir quelque chose de la personnalité de l'acteur", ce qui implique une importante relation entre les personnages et les comédiens dont la conséquence, sur scène, est la manifestation de leurs singularités: "ils sont tous, volontairement, chacun un monde", l'enjeu étant la confrontation de ces mondes particuliers.

C'est ainsi, plus précisément, que les comédiens montrent au public des différences d'intention ou de motivation dans leur façon de dire leur texte, et ce très concrètement. Jouée toujours par une brésilienne, le personnage de Madame Queuleu se remarque d'abord dans sa grande expressivité et l'humour qu'elle cherche à déclencher: presque toujours prise au sein des conflits entre Adrien et Mathilde, elle n'hésite pas à exagérer son énervement. Florence Dupont, dans sa communication ${ }^{4}$ au Colloque Koltès, se demandait si Le Retour au désert commence comme une comédie de boulevard. Non seulement la mise en scène de Catherine Marnas prend acte d'éléments qui dans le texte original peuvent conduire à ce genre mais, dans le spectacle, ce genre tient presque tout entier dans le personnage de Madame Queuleu dont le paroxysme se situe dans la dernière scène où elle crie avec effroi: "Noirs, monsieur, ils sont tout noirs...”* Si chaque comédien manifeste ainsi sa singularité, il s'ensuit qu'ils incarnent chacun un monde théâtral coexistant à celui des autres acteurs. Ainsi le personnage de Mathieu, joué à la fois par un acteur brésilien qui exploite un jeu plus caricatural, manifesté par sa gestuelle particulièrement ample, et par un acteur français qui, lui, exploite un registre plus ironique, cynique, dû à la posture nonchalante qui le caractérise. S'il y a ainsi confrontation de mondes, ceux propres à chaque comédien, cela donne sur scène une confrontation de genres théâtraux - ou de registres - qui semblent à certains moments intrinsèques à l'écriture de Koltès - par exemple, dans la scène plutôt burlesque de réunion de Sablon, Plantières et Borny. À cette énumération de genres on pourrait ajouter également, pour peu que cela soit un genre, une dimension plus performative de la pièce de Catherine Marnas, et ce, dès le départ, alors que les spectateurs s'installent et que les comédiens attendent sur une scène éclairée le retour de Mathilde et le début de la pièce. On retrouve également cette dimension toutes les fois où le texte est dit au micro, mettant en exergue l'acte locutoire et la sonorité de la parole.

\footnotetext{
${ }^{4}$ Intitulée "Le Retour au désert commence-t-il comme un vaudeville?"
} 
Plus généralement, cette dimension performative va de pair, semble-t-il, avec une dynamique théâtrale conçue par la coexistence et l'affrontement sur scène d'éléments hétérogènes et de singularités, exposant la métathéâtralité de la pièce. En ce sens, il est particulièrement frappant de considérer le mélange des langues que l'on trouve déjà dans le texte original, puisqu'aux première et quinzième scènes les personnages de Mathilde, Aziz et Saïfi parlent à la fois en arabe et en français. Dans sa mise en scène, Catherine Marnas généralise ce premier bilinguisme et en fait un véritable dispositif dramaturgique. Comme on l'a déjà rapidement dit, ce Retour au désert fait coexister la version originale et française du texte ainsi que sa traduction en portugais du Brésil. La plupart du temps sont mises ainsi côte à côte, dans une même scène, des répliques en chacune de ces deux langues non exclusives à tel ou tel comédien ou à tel à personnage. Ce bilinguisme se localise même de nombreuses fois dans une seule et même réplique où l'on passe ainsi entre les langues presqu'indifféremment du point de vue des comédiens qui les emploient d'une seule traite. D'abord, il y a des effets de sens: Madame Queuleu, peut-on remarquer, s'exprime en français lorsqu'elle s'adresse aux maîtres de maison et en portugais lorsqu'elle s'adresse à Aziz, par exemple, montrant par là une rupture sociale en jeu dans l'emploi des langues. Autre exemple, dans la scène 3, le personnage de Fatima, jouée par une brésilienne, s'adresse à sa mère en portugais puis en français face à Mathieu lorsqu'elle veut justement maintenir la distance. On peut voir un premier enjeu de sens, un enjeu dramaturgique, à déployer ainsi un bilinguisme dont on verra qu'il n'est pas si systématique. Celui-ci a comme conséquence particulière de mettre côte à côte plusieurs postures du texte-source de Koltès: une posture d'abord familière, pour le public français, lorsque le texte est dit dans la langue originale, une posture textuelle lorsque celui-ci est projeté sur les murs de la scénographie pour traduire les répliques dites en portugais, cette langue et cette version correspondant à leur tour à une posture exotique du texte. Celle-ci apporte enfin, toujours au public francophone, des nouveautés du langage qui se manifestent par exemple par les différences d'accentuation, très nettes entre le français et le portugais du Brésil. Il s'agit même d'y voir l'apport d'une version du texte qui se reçoit alors dans sa sonorité, sa musicalité, le sens étant relégué au texte projeté sur les murs. Parfois ces postures se croisent, principalement lorsque le texte original est dit par 
- (KOLTÈS, Bernard-Marie. Le Retour au désert. op. cit.: 57.)

- (GLISSANT, Edouard. Traité du tout-monde. Paris: Gallimard, 1997: 22.)

(KOLTÈS, Bernard-Marie. Lettre à Hubert Gignoux du 7 avril 1970. In: Lettres. op. cit.: 115.) un comédien brésilien et que la langue française se trouve subtilement altérée, singularisée, par un accent étranger. C'est ainsi que plus généralement la mise en scène de Catherine Marnas, par cette interpénétration des langues et des langages, cherche à mettre en œuvre de la manière la plus directe qui soit l'affrontement de mondes différents mais surtout leur altération réciproque.

De cette manière, il s'agit pour cette mise en scène de prendre acte du monde actuel, comme le faisait Koltès dans ses pièces, et de l'apporter sur un plateau. Un monde dont les frontières sont traversées de toutes parts et par ce biais au minimum problématisées et questionnées. La mise en œuvre de la création du Retour au désert manifeste d'ailleurs déjà, du moins peut-on l'imaginer par le constat de sa franco-brésilianité, son inscription dans une dynamique politique qui consiste à traverser incessamment les frontières du monde - en l'occurrence celles de la France et du Brésil. C'est ainsi que ce projet de mise en scène se trouve d'emblée dans une mondialisation qu'il partage avec les époques que Koltès confronte dans Le Retour au désert, les années 60 et les années 80. Aussi trouve-t-on son expression par exemple dans la réplique du parachutiste noir:

On me dit que les frontières bougent comme la crête des vagues, mais meurt-on pour le mouvement des vagues? On me dit qu'une nation existe et puis n'existe plus, qu'un homme trouve sa place et puis la perd, que les noms des villes, et des domaines, et des maisons, et des gens dans les maisons changent dans le cours d'une vie, et alors tout est remis en un autre ordre et plus personne ne sait son nom, ni où est sa maison, ni son pays, ni ses frontières. Il ne sait plus ce qu'il doit garder. Il ne sait plus qui est l'étranger.

Si ce passage décrit bien à la fois le trouble du monde qui se manifeste dans le microcosme d'Adrien et Mathilde et celui qui se manifestait à l'écrivain, aujourd'hui il apparaît de manière évidente dans l'actualité. Edouard Glissant parle dans Traité du toutmonde d'un "chaos-monde" qui désigne "le choc actuel de tant de cultures qui s'embrasent, se repoussent, disparaissent, subsistent pourtant, s'endorment ou se transforment, lentement ou à vitesse foudroyante."* Face au constat d'un tel choc qui rappelle de loin la lettre de Koltès à Hubert Gignoux lorsque celui-ci parle des rapports entre les personnes ou les personnages comme de "deux bateaux posés chacun sur deux mers en tempête [...] le choc dépassant de loin la puissance des moteurs", Edouard Glissant implique ce qu'il appelle une 
Poétique de la Relation, ce possible de l'imaginaire qui nous porte à concevoir la globalité insaisissable d'un tel Chaos-monde, en même temps qu'il nous permet d'en relever quelque détail, et en particulier de chanter notre lieu, insondable et irréversible.

L'écriture koltésienne, comme elle se présente en tout cas dans Le Retour au désert, trouverait bien dans cette citation l'explicitation de son rapport au monde, correspondant à la réplique du parachutiste noir qui juxtapose lui aussi le global au particulier dans la réplique précédemment citée, au sein de laquelle sont mis sur un même plan la nation, la place, la ville, le domaine, la maison, le nom et le pays dont les frontières ne cessent de bouger. La problématique territoriale comme exprimée par ces deux auteurs correspond à une dynamique des personnages du Retour au désert dont les conflits sont tout d'abord liés à leur relation au lieu propre et à leur identité.

Ce fait dramatique, mais aussi ce fait d'actualité, le Retour au désert de Catherine Marnas en prend acte dans sa mise en scène qui s'ancre d'emblée de nos jours par les costumes actuels dans lesquels les personnages se présentent. Sa scénographie repose sur la présence mobile de deux murs angulaires qui dépassent en hauteur les comédiens et qui à eux seuls servent à indiquer les différents lieux dans lesquels se passe l'action. Il est notable que dans le texte les didascalies qui les indiquent se réferent aux limites physiques de la maison: "mur qui entoure le jardin"; " "couloir"; "le mur d'enceinte"* et, en cela, à des territoires-frontières entre le dehors et le dedans. D'autres didascalies viennent renforcer l'aspect ambigu de territoires faillibles par ces portes entrouvertes, en scène 1 et en scène 5 , ou bien qui sont à la fois intérieures et extérieures comme le "jardin"” ou la "véranda". "Catherine Marnas représente cet aspect paradoxal de ces lieux dramatiques par ces deux murs qui eux aussi, même s'ils délimitent un territoire du drame, présentent toujours des sorties ou des entrées vers son extérieur et donc vers le reste du plateau. Et ce à partir de la première scène, particulièrement emblématique de cette dramatisation de la scène. En effet, au départ, les deux murs d'angles sont encastrés l'un dans l'autre, de sorte que le public n'en voit qu'un seul et que le passage entre les deux, intérieurs, n'est que deviné par les déplacements de Madame Queuleu - le bloc ainsi formé représentant le territoire de la maison. À l'arrivée de Mathilde, correspond alors le premier changement de place de ces murs, de manière significative. Ainsi, immédiatement
*(GLISSANT, Edouard. Traité du tout-monde. op. cit.: 22.)

(KOLTÈS, Bernard-Marie. Le Retour au désert. op. cit.: 11.)

* (Ibidem: 27.)

( (Ibidem: 51.)

" (Ibidem: 21,43,74.)

"(Ibidem: 54.) 
après s'être présentée, Mathilde pousse le premier mur visible vers le fond de la scène, entrainnant, avec l'aide d'autres comédiens, le déplacement de l'autre mur de sorte qu'ils seront dans leur position finale l'un à côté de l'autre créant un espace interstitiel entre eux, comme un couloir, fuyant vers le lointain de la scène. Ce déplacement spectaculaire, d'autant plus qu'il est accompagné d'une musique de tango, ouvre ainsi le plateau et manifeste l'ouverture critique d'un territoire qui ne cessera de se modifier par la suite. $\mathrm{Si}$ les mouvements de ces murs sur le plateau sont la représentation dramatique de problématiques territoriales dans Le Retour au désert, de même il s'agit de représenter la relation des personnages avec ces limites et l'enfermement qui en découle: Mathieu qui entend sauter par-dessus les murs, Mathilde qui les déstabilise en s'y introduisant, les voix des discordes dont Madame Queuleu dit qu'ils "traversent les murs", " l'intrusion du parachutiste noir derrière Adrien ou bien la libération des limites terrestres d'Edouard qui disparait d'un coup, en sautant. La forme territoriale est ainsi déstabilisée de toute part et sa représentation par les deux murs mobiles porte à la fois l'enjeu dramatique du Retour au désert mais aussi un enjeu politique, justement en rapport avec l'actualité du Brésil. En effet, l'enfermement dans cette maison fait fortement écho à l'organisation des mégalopoles dans ce pays où les multiples résidences ou villas sont surveillées par des caméras, des hélicoptères même, dont on entend le bruit au début du spectacle, et sont entourées de murs empêchant toute intrusion: ils sont ainsi coupés de l'extérieur. Si cet aspect actuel est plus frappant pour un public brésilien, il s'agit aussi de voir dans ces murs une rupture plus symbolique avec l'étranger propre peut-être plus particulièrement au pays français et sa relation, par exemple, avec les immigrés; il s'agit en tous les cas d'une rupture avec l'autre, qu'il soit d'un autre pays ou d'une autre classe sociale.

Cette actualité montre bien les résonances possibles du Retour au désert avec le monde actuel, notamment encore dans sa partition nord/sud. Et en montrant les troubles portés aux territoires, il s'agit également d'en montrer les conséquences pour les individus qui y sont liés. Mathilde est ainsi emblématique d'une identité sans aucune attache territoriale: elle dit ainsi très clairement, dès la deuxième scène: "mes racines? Quelles racines? Je ne suis pas une salade". Aziz, quant à lui, exprime le doute sur son identité territoriale comme suit, dans la scène 15: "Le Front dit que je suis un 
Arabe, mon patron dit que je suis domestique, le service militaire dit que je suis français, et moi, je dis que je suis un couillon". Le trouble, on peut le voir, vient d'une multiplicité des possibles identitaires au sein d'une seule et même personne. Catherine Marnas dans son dispositif théâtral concrétise ainsi scéniquement cette multiplicité identitaire. En effet, les personnages de Mathieu, Mathilde et Adrien, personnages qui s'affrontent de manière particulièrement sensible à l'ailleurs et à l'autre, sont systématiquement joués par deux acteurs, doublés donc, l'un brésilien et l'autre français, qui se partagent de manière non systématique les répliques du texte. On peut repérer cependant trois types de moment: ceux où l'un des acteurs répète en portugais ce que l'autre disait en français, ceux où les acteurs se complètent et continuent la même réplique, ceux où ils parlent ensemble, presque d'une seule voix. La relation de ces duos par rapport au texte est évidemment à chaque fois modulée en fonction de la scène et des effets de sens que cela produit. Ainsi, pour la scène 6 , Adrien et Mathilde sont formés chacun de trois comédiens qui arrivent au fur et à mesure que s'intensifie le conflit entre ces deux personnages. Les deux trios s'affrontent d'abord par la parole, en français, et les répliques sont dites successivement par chacun des comédiens. Et à des moments cruciaux, ils les disent tous d'une seule voix, relevant certaines parties du texte, notamment dans cette scène les pronoms possessifs. Le trouble identitaire est ainsi manifesté par l'instabilité de ces personnages due à leur dédoublement, aux deux langues qu'ils emploient mais aussi à leur posture physique toujours localisée dans la pulsion du déplacement, et surtout à la relation qui se joue entre les comédiens jouant un même personnage par leurs réactions réciproques à leur altérité incluse.

Si la mise en scène vue depuis ses particularités peut alors apparaître dans des ruptures qu'elle met en place (entre les langues, les manières de dire et entre les membres des duos), le spectacle offre au contraire par ces relations une dynamique théâtrale qui s'appuie sur une coexistence d'hétérogénéités et qui fait jouer ces ruptures de telle sorte que les personnages parlent d'un même souffle et que le spectacle se déroule dans une même énergie scénique. Il s'agit de manière très générale de caractériser ainsi la cohérence de la mise en scène qui semble ainsi répondre sur scène au "chaosmonde" qu'elle exploite. D'abord par une dynamique d'altération qui se joue entre les différentes postures du texte, des personnages, 
des langues, du théâtre et des mondes qu'ils manifestent chacun. L'altération porte ainsi plus généralement sur la forme d'identité, toujours la même et toujours une, qui permettrait de caractériser unilatéralement ces différents éléments. Bien au contraire, Catherine Marnas propose de les déstabiliser en multipliant la forme de départ et en impliquant, par une altération au moins bilatérale, l'évolution incessante de ces formes. La scène est bien à ce titre le territoire qui accuse cette énergie par la présence de ces murs qui ne cessent de se déplacer et de le présenter à chaque fois sous une posture différente, altéré donc. Les comédiens, quant à eux, sont ceux qui opèrent ces déplacements scénographiques et plus généralement ce sont eux aussi qui soutiennent et manifestent cette énergie théâtrale qui prend la pièce en deçà de ses ruptures et différences intrinsèques. Une énergie due bien sûr au travail de mise en scène et de direction de Catherine Marnas, qui cherche à animer les personnages de ce qu'elle appelle une "force vitale" qui correspondrait ainsi à la dynamique d'altération généralisée de ce spectacle. Cette dynamique, on la devine plus particulièrement par l'expression de "l'âme de fond" que le metteur en scène utilise pour décrire plus précisément cette force vitale. Reprenant la lettre de Koltès à Hubert Gignoux, il s'agit d'actualiser ainsi une conception koltésienne de personnages qui seraient animés d'abord par une énergie spirituelle, par une "âme de fond", mais aussi par des mouvements incessants, ceux de la mer, qui néanmoins les tirent vers telle ou telle direction. La mise en scène prend acte de cette force ou de cette énergie en proposant bien sûr des allers et des retours entre plusieurs postures d'une même pièce qui avance et qui se déroule par ces va-et-vient qui lui sont propres, et toujours en direction de l'autre.

Cet autre, bien sûr, étant le public de ce spectacle puisque c'est lui en dernier lieu qui a la charge d'accuser et de recevoir cette énergie d'altération. C'est par lui que passe d'abord une différenciation entre le même et l'autre, puis leur mise en relation forcée par le dispositif théâtral qui questionne ainsi ses éléments constitutifs, surtout ses personnages et son texte. Ils sont aussi les opérateurs des actualisations du texte-source de Koltès proposées par cette coïncidence théâtrale de plusieurs différends "et alors tout est remis en un autre ordre et plus personne ne sait son nom, ni

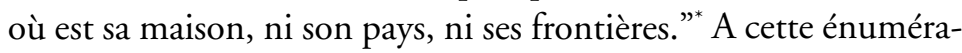
tion, il faudrait ajouter ce territoire toujours autre, la scène, tou- 
jours recréée, réinventée, qui prend acte de sa posture de monde et au monde en croisant quelques-unes de ses parties, quelques-unes de ces actualités, dans cette mise en scène qui ainsi se confronte à Koltès, maintenant.

\section{Guillaume Pinçon}

Doutorando sob regime de cotutela internacional orientado pelo professor José da Costa na UNIRIO e pelo professor Christophe Bident na Universidade Paris 7 Denis Diderot. Desenvolve uma tese sobre a noção de teatralidade a partir de estéticas teatrais da Cia. dos Atores, do Teatro Oficina, do Teatro da Vertigem e do grupo Lume.

\section{Resumo}

Pela análise da encenação de Catherine Marnas de Retorno ao deserto, peça escrita por Bernard-Marie Koltès, trata-se de investigar os diálogos possíveis entre a obra do autor e o mundo contemporâneo destacando no espetáculo elementos que fazem vir à tona a problemática política da relação com o outro, com o estrangeiro, com o território.

Abstract

Catherine Marnas's production of Return to the desert which was written by BernardMarie Koltès - aims at looking for possible exchanges between the author's work and the contemporary world, by stressing elements that emphasize political issues of relationship concerning the other, the stranger, and territory.

\section{Résumé}

Par l'analyse de la mise en scène de Catherine Marnas du Retour au désert, pièce écrite par Bernard-Marie Koltès, il s'agit de chercher les dialogues possibles entre l'œuvre de l'auteur et le monde contemporain en relevant dans le spectacle des éléments qui mettent en relief les problématiques politiques de relation à l'autre, à l'étranger, au territoire.
Palavras-chave: teatro; contemporâneo; franco-brasileiro; bilinguismo; território.

Key words: theatre; contemporary; Franco-Brazilian; bilingualism; territory.

Mots-clés: théâtre; contemporain; franco-brésilien; bilinguisme; territoire. 